\title{
QUANTIZATION WITH ADAPTATION - ESTIMATION OF GAUSSIAN LINEAR MODELS*
}

\author{
LÁSZLÓ GERENCSÉR ${ }^{\dagger}$, ILDIKÓ KMECS ${ }^{\ddagger}$, AND BALÁZS TORMA $§$
}

\begin{abstract}
Quantization is a basic operation in communication, having a considerable impact also on control, in particular on control over communication networks, see [2] for an early reference. In this paper we consider a classic, seemingly innocent problem of reconstructing a single signal value $\theta^{*}$ when measured with additive Gaussian noise, followed by uniform quantization of sensitivity $h$, with or without saturation. A peculiar feature of the above estimation problem is that its Fisher information varies considerably with the noise variance and the location of the true parameter. It is therefore a meaningful objective to adjust (shift) the quantization levels so as to maximize the Fisher information or to inject additional measurement noise for the same purpose. We shall focus on the first problem. Empirical evidence shows that, for given noise variance, the Fisher information is maximal when the location parameter is of the form $\theta^{*}=k h+h / 2$. Adjusting the quantization levels is equivalent, from the statistical point of view, to adjusting, say increasing the location parameter by an amount of $\delta>0$ to achieve a known target, say $\eta^{*}=k h+h / 2$ for some integer $k$. The problem that we address in this paper is if such an adjustment of the problem can be done adaptively, in the context of a previously developed recursive, real-time estimation method for estimating $\theta^{*}$, that was called a randomized $E M$-method for estimating $\theta^{*}$. We give a positive answer to this question. The proposed method results in considerable improvement in efficiency, supported both by the algebra of the asymptotic theory of stochastic approximation, and by extensive experimental evidence. The basic ideas developed and presented for this benchmark problem can be easily generalized for the multi-variable case.
\end{abstract}

Keywords: quantization; Gaussian linear regression; EM-method; Metropolis-Hastings method; stochastic approximation.

1. Introduction. Quantization is a basic operation in communications, arising among others in analog-to-digital conversion or in data-compression, having a considerable impact also on control, in particular on control over communication networks. The latter application area is particularly fit for the present issue honoring Roger Brockett, due to his fundamental contribution to the area in his 1998 paper, (coauthored by D. Liberzon), [2]. See also [14] for a recent survey on quantization in communication and control.

A scalar quantizer is defined as a mapping $q$ from $\mathbb{R}$ to a discrete, finite or countable set $\mathcal{Y} \subset \mathbb{R}$, representing the so-called quantization levels, assigning to each

\footnotetext{
*Dedicated to Roger Brockett on the occasion of his 70th birthday.

$\dagger$ Corresponding author, MTA SZTAKI, 13-17 Kende u., Budapest 1111, Hungary.

E-mail: gerencser@sztaki.hu, Tel: (36-1)-279-6138, (36-1)-279-6190, Fax: (36-1)-466-7503.

${ }^{\ddagger}$ SIEMENS PSE KFT, 51-57 Gizella üt, Budapest 1143, Hungary.

E-mail: ildiko.kmecs@siemens.com, Tel: (36-1)-471-3576.

$\S$ MTA SZTAKI, 13-17 Kende u., Budapest 1111, Hungary.

E-mail: torma@sztaki.hu, Tel: (36-1)-279-6158.
} 
$x \in \mathbb{R}$ its quantized version

$$
y=q(x) .
$$

The simplest scalar quantizer is the uniform quantizer, where the set of quantization levels is given by the integer multiples of a fixed, positive number, say $h$, called the sensitivity of the quantizer, and if $x$ is a real number then we set

$$
q(x)=k h \quad \text { for } \quad I_{k}=\{k h-h / 2<x \leq k h+h / 2\} .
$$

A more realistic model for quantization is a quantizer with saturation, see [2], defined as above in the range

$$
-(M+1 / 2) h<x \leq(M+1 / 2) h,
$$

with $M$ being a positive integer, and setting $q(x)= \pm M h$ outside the above interval. Thus there are altogether $2 M+3$ quantization domains, and we will denote them again by $I_{k}$, with $k \in K$, where $K$ is the set of possible indices. In this paper we consider a classic, seemingly innocent problem of reconstructing a single signal value $\theta^{*}$ when measured with additive Gaussian noise, followed by quantization. I.e. the observed values are of the form

$$
y_{n}=q\left(\theta^{*}+e_{n}\right),
$$

where $e_{n}$ is an i.i.d. Gaussian sequence with mean 0 and known variance $\sigma^{2}=\left(\sigma^{*}\right)^{2}$, see e.g. [10]. The assumed knowledge of $\sigma^{2}$ may be unrealistic in many applications, but it greatly simplifies the presentation. We shall discuss the possibility of handling unknown $\sigma$-s at the end of the paper. One of the motivations for revisiting this problem is the current interest in mobile communications to reduce the required resources such as power or channel capacity while retaining the quality of service.

A peculiar feature of the above estimation problem is that its $2 \times 2$ Fisher information matrix varies considerably with the noise variance and the location of the true parameter. We have empirical evidence of the fact that the off-diagonal elements of the Fisher information matrix, say $R^{*}$ are zero. Thus the asymptotic covariance of the ML estimator of $\theta^{*}$ will be equal to the inverse of the $(1,1)$ element of the Fisher information matrix, denoted by $r^{*}=r_{11}^{*}$. For brevity sake we call this the Fisher information for $\theta^{*}$. It is an empirical fact, that $r^{*}$ is small for small $\sigma^{*}$, since then quantization has a scrambling effect. These features are exhibited on the figures below. In all experiments we assume $h=1$, and $M=10$. On Figure 1 on the left we plot the Fisher information against the variance when $\theta^{*}=0$ and $\theta^{*}=0.5$, respectively. On the right we plot the Fisher information against the location parameter, varying between 0 and 1 , when the variances are $\left(\sigma^{*}\right)^{2}=0.08$ and $\left(\sigma^{*}\right)^{2}=0.1$, respectively.

It is therefore a meaningful objective to adjust (shift) the quantization levels so as to maximize the Fisher information or to inject additional measurement noise for 

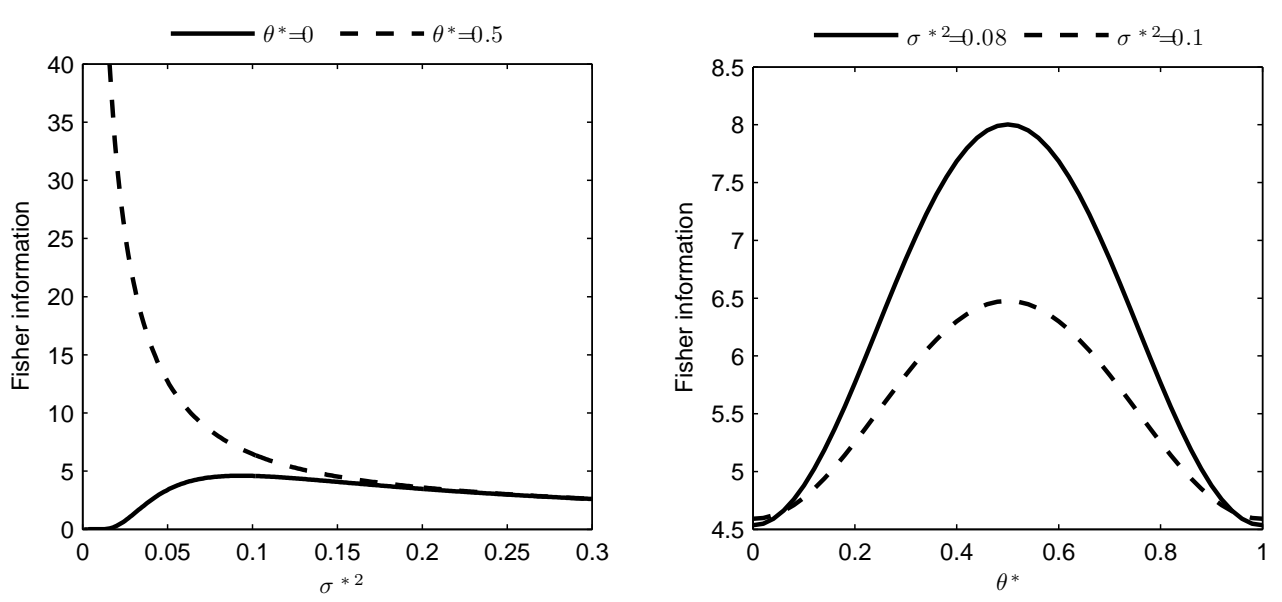

FIG. 1. The Fisher information with respect to $\theta$.

the same purpose. We shall focus on the first problem. It is seen from the figures that the Fisher information is maximal when the location parameter is of the form $\theta^{*}=k h+h / 2$. The Fisher information has a large variation in $\theta^{*}$ if the variance is small (see Fig. 1 on the left). For example, for $\left(\sigma^{*}\right)^{2}=0.01$ it varies between 0.0015 and 63.662 , for $\left(\sigma^{*}\right)^{2}=0.3$ it spans the interval between 2.6051 and 2.6154. Adjusting the quantization levels is equivalent, from the statistical point of view, to adjusting, say increasing the location parameter by an amount of $\delta>0$. If $\theta^{*}$ stands for, say, a very small weight of a physical object, then an increase can indeed be realized by adding a known weight to achieve a known target, say $\eta^{*}=h / 2$. The problem that we address in this paper is if such a shifting can be done adaptively, using observations of the form, with known $\delta$ :

$$
y_{n}=q\left(\theta^{*}+\delta+e_{n}\right) .
$$

An efficient randomized $E M$-method to solve the off-line maximum-likelihood estimation problem, based on say $N$ observations, has been developed in [4]. In the course of this procedure we generate a sequence of estimators $\theta_{t}$ that converge to the off-line maximum likelihood estimator $\hat{\theta}_{N}$ almost surely, under appropriate technical conditions. A real-time version of this method, exhibiting excellent convergence properties, has been developed in [5]. In the real-time scheme we generate a sequence of estimators $\hat{\theta}_{t}$ such that $\hat{\theta}_{t}$ converges to $\theta^{*}$ almost surely, under appropriate technical conditions. The asymptotic covariance of $\hat{\theta}_{t}$ can be expressed as the solution of a Lyapunov-equation, using the algebra of Theorem 13, Chapter 4.5.3, Part II of [1]. It is well-known from the theory of stochastic approximation, that, in the case of a weighted stochastic gradient method based on the maximum-likelihood estimation, the best available asymptotic covariance is the inverse of the Fisher information. This is achieved by a stochastic Newton-method. 
Taking into account the volatility of the Fisher information as a function of $\theta^{*}$, it makes sense to try to improve the efficiency of our real-time randomized $E M$-method by adjusting the problem so that $\theta^{*}$ is in fact equal to a quantization level. We will show that such an adaptive adjustment is indeed possible, resulting in considerable improvement in efficiency. The latter will demonstrated by the results of extensive experimental studies. The basic ideas developed and presented for the above benchmark problem can be generalized for the multi-variable case.

The theoretical analysis of the method can be carried out with the theory of recursive estimation under Markovian dynamics developed in [1, 11]. Although this theory is not complete, inasmuch a basic problem, the possibility of the estimator leaving any fixed compact domain is treated in a practically unsatisfactory manner, this deficiency can be rectified, see [8].

The presentation and verification of all conditions required by the BMP theory would exceed the allotted space. Henceforth we will restrict ourselves to the verification of a key condition of the BMP theory, namely the stability of the associated ODE, see Chapter 1.5, Part II of [1], and the computation of the asymptotic covariance matrices of the relevant estimators, using the algebra of Theorem 13, Chapter 4.5.3, Part II of [1].

2. The $E M$-method for estimating $\theta^{*}$. Consider first the case of off-line estimation, i.e. when the number of samples $N$ is fixed. For each $y$ in the observation set define the quantization domain $I(y)=\{x: q(x)=y\}$. Write as usual

$$
\phi\left(x ; \theta, \sigma^{2}\right)=\frac{1}{\sqrt{2 \pi} \sigma} e^{-\frac{(x-\theta)^{2}}{2 \sigma^{2}}} .
$$

Then for any $\theta$ the $\theta$-probability of observing $y$ is, with $\sigma^{2}=\left(\sigma^{*}\right)^{2}$,

$$
P(I(y) ; \theta)=\int_{I(y)} \frac{1}{\sqrt{2 \pi} \sigma} e^{-\frac{(x-\theta)^{2}}{2 \sigma^{2}}} d x=\int_{I(y)} \phi\left(x ; \theta, \sigma^{2}\right) d x .
$$

For any $\theta$ the logarithm of the $\theta$-probability of observing $y^{N}=\left(y_{1}, \ldots, y_{N}\right)$ is

$$
L_{N}\left(y^{N} ; \theta\right)=\sum_{n=1}^{N} \log P\left(I\left(y_{n}\right) ; \theta\right)=\sum_{n=1}^{N} L\left(y_{n} ; \theta\right) .
$$

In Figure 2 we plot the expected likelihood function against the running parameter $\theta$, with $\sigma$ kept fixed at $\sigma^{*}$ (left), and against the running parameter $\sigma$ with $\theta$ kept fixed at $\theta^{*}$ (right). Two problems are considered: a well-conditioned problem (solid line) with parameters

$$
\theta^{*}=0.5, \quad \sigma^{*^{2}}=0.08,
$$


and an ill-conditioned problem (dashed line) with parameters

$$
\theta^{*}=0, \quad \sigma^{*^{2}}=0.1 \text {. }
$$

According to these figures the expected likelihood function is likely to be concave with unique maximum. It is also seen from the figure on the right, that the expected loglikelihood function is quite flat in $\sigma$ in the case of the problem that is well-conditioned for estimating the location parameter $\theta^{*}$.
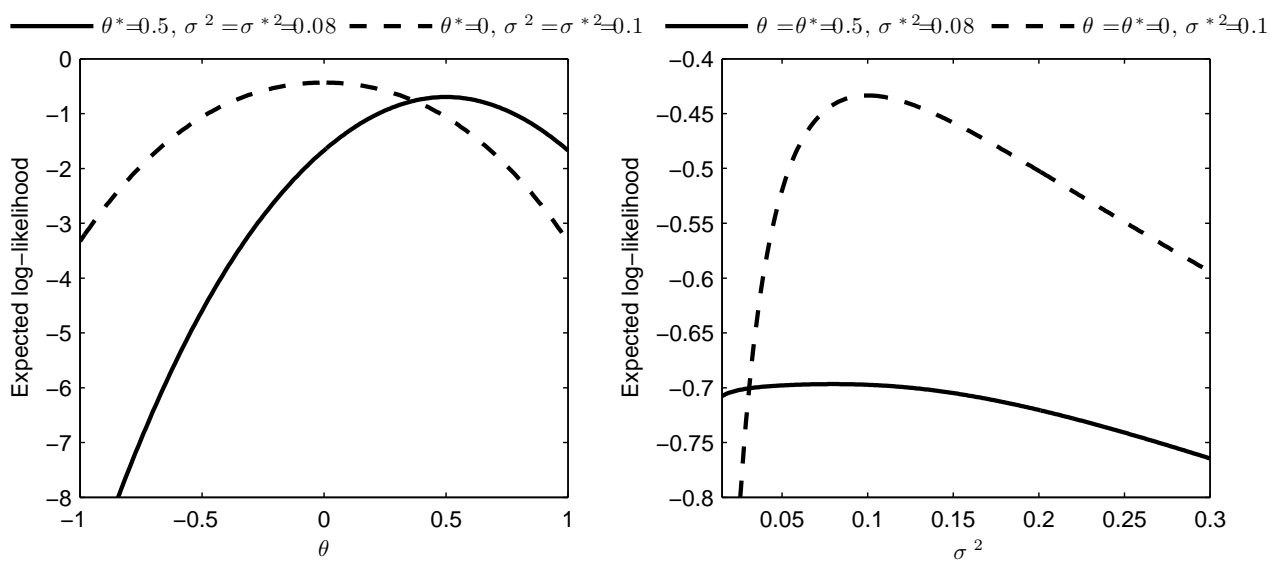

FIG. 2. The expected log-likelihood function.

The ML estimator $\hat{\theta}_{N}$ is obtained by maximizing $L_{N}\left(y^{N} ; \theta\right)$, or solving the likelihood equation

$$
\frac{d}{d \theta} L_{N}\left(y^{N} ; \theta\right)=0
$$

Introducing the conditional density of $x$ given $y$

$$
\phi\left(x \mid y ; \theta, \sigma^{2}\right)=\frac{\phi\left(x ; \theta, \sigma^{2}\right)}{P(I(y) ; \theta)} \chi_{I(y)}(x),
$$

where $\chi_{E}(x)$ denotes the indicator of the set $E$, the likelihood equation is equivalent to the following:

The quantized normal equation:

$$
\left(\sum_{n=1}^{N} \int \phi\left(x \mid y_{n} ; \theta, \sigma^{2}\right) d x\right) \theta=\sum_{n=1}^{N} \int x \phi\left(x \mid y_{n} ; \theta, \sigma^{2}\right) d x .
$$

Notice that this equation is non-linear in $\theta$. To solve the likelihood equation would require the computation of an integral in each step of the iteration, which is not feasible if $\theta^{*}$ is vector-valued.

The EM-method: This difficulty has been circumvented in [4] by using a Markov Chain Monte Carlo $(M C M C)$ method for computing the integrals. Since the likelihood of the observations $x_{n}=\theta^{*}+e_{n}$ is easily obtained, and $y_{n}=q\left(x_{n}\right)$, a natural 
approach to solve the likelihood equation is to use the $E M$-method. Following the basic steps of the $E M$-method we replace the log-likelihood function by an auxiliary function (see e.g. [3])

$$
Q(y ; \theta, \bar{\theta})=\mathrm{E}_{\bar{\theta}}\left[\log p\left(X, \theta, \sigma^{2}\right) \mid y\right]=\mathrm{E}\left[\log p\left(X, \theta, \sigma^{2}\right) \mid y ; \bar{\theta}\right],
$$

where $\bar{\theta}$ is a current best estimate, and the random variable $X=\bar{\theta}+e$ is the unknown assumed state. For $N$ independent observations we set

$$
Q_{N}\left(y^{N} ; \theta, \bar{\theta}\right)=\sum_{n=1}^{N} Q\left(y_{n} ; \theta, \bar{\theta}\right)=\mathrm{E}\left[\log p\left(X^{N}, \theta\right) \mid y^{N} ; \bar{\theta}\right],
$$

where $X_{n}=\bar{\theta}+e_{n}$ is the unknown assumed state at time $n$. The so-called $M$-step, maximizing $Q_{N}$ in $\theta$, gives an updated estimate that will replace $\bar{\theta}$.

To simplify the notations consider now the case of uniform quantization without saturation. Let $I_{k}$ be the $k$-th interval: $I_{k}=\{x: q(x)=k h\}$, and let

$$
N_{k}=\#\left\{n: 1 \leq n \leq N, y_{n} \in I_{k}\right\}
$$

be the number of times that $k h$ is observed in the sequence $y^{N}$. Then the $M$-step is equivalent to solving the linear equation

$$
\frac{d}{d \theta} Q_{N}\left(y^{N} ; \theta, \bar{\theta}\right)=\sum_{k} N_{k} \int_{I_{k}} \frac{(x-\theta)}{\sigma^{2}} \phi\left(x \mid k h ; \bar{\theta}, \sigma^{2}\right) d x=0 .
$$

Note that all information on the data is now contained in the counting numbers $N_{k}$. Thus we arrive at the following updating formula:

The $M$-step:

$$
\theta=\sum_{k \in K} \frac{N_{k}}{N} \int_{I_{k}} x \phi\left(x \mid k h ; \bar{\theta}, \sigma^{2}\right) d x .
$$

In the course of the $E M$-method we set $\bar{\theta}=\theta_{t}$, and we get $\theta=\theta_{t+1}$.

Basic inequalities. The basic inequality connecting the likelihood function and the $Q$-function is the following: for any $y$ and for given fixed $\bar{\theta}$ we have for any $\theta$

$$
L(y, \theta) \geq Q(y ; \theta, \bar{\theta})+D(\bar{\theta} \| \theta)+H(\bar{\theta}),
$$

where $D(\bar{\theta}|| \theta) \geq 0$ for all $\theta$. (In fact $D(\bar{\theta} \| \theta)$ is a divergence between two conditional probability densities, and $H(\bar{\theta})$ is an entropy, which depends only on $\bar{\theta}$.) It follows that the function $L(y, \theta)-Q(y ; \theta, \bar{\theta})$ is minimized at $\theta=\bar{\theta}$, thus, if $\bar{\theta}$ is interior relative to the the parameter domain then, we have for any $N$ and $y^{N}$

$$
Q_{N}^{\prime}\left(y^{N} ; \theta, \theta\right)=\frac{\partial}{\partial \theta} Q_{N}\left(y^{N} ; \theta, \bar{\theta}\right)_{\mid \bar{\theta}=\theta}=\frac{d}{d \theta} L(y ; \theta) .
$$


It follows that the solution of the likelihood equation $\frac{d}{d \theta} L_{N}\left(y^{N} ; \theta\right)=0$ is obtained by solving the equation

$$
Q_{N}^{\prime}\left(y^{N} ; \theta, \theta\right)=0
$$

Let us now consider the asymptotic log-likelihood function

$$
\bar{L}(\theta)=\mathrm{E}_{\theta^{*}} \log P(I(Y) ; \theta) .
$$

Here $Y=q\left(\theta^{*}+e\right)$. It is well-known and directly seen that

$$
\frac{d}{d \theta} \bar{L}(\theta)_{\mid \theta=\theta^{*}}=0 .
$$

Similarly, define the asymptotic $Q$-function, with $X=\theta^{*}+e$ and $Y=q(X)$, as

$$
\bar{Q}(\theta, \bar{\theta})=\mathrm{E}[\mathrm{E}[\log P(X, \theta) \mid Y, \bar{\theta}]],
$$

where the outer expectation is $\mathrm{E}_{\theta^{*}}$. To relate the asymptotic $Q$ function to the asymptotic likelihood function the simplest, although formal, procedure is to divide both sides of (16) by $N$, and take limit to get

$$
\bar{Q}^{\prime}(\theta, \theta)=\frac{\partial}{\partial \theta} \bar{Q}(\theta, \bar{\theta})_{\mid \bar{\theta}=\theta}=\frac{d}{d \theta} \bar{L}(\theta) .
$$

Thus the asymptotic problem of determining $\theta^{*}$ can be formulated, as solving the equation

$$
\bar{Q}^{\prime}(\theta, \theta)=0
$$

This equation could be derived directly, but the context of the EM-method gives a convenient computational framework that will be exploited subsequently.

The Fisher information. The Fisher information for the problem of estimating $\theta^{*}$ with known $\sigma=\sigma^{*}$ will be denoted by

$$
r^{*}=-\left.\frac{d^{2}}{d \theta^{2}} \bar{L}(\theta)\right|_{\theta=\theta^{*}}
$$

It is well-known that it can be expressed via the score function

$$
\frac{d}{d \theta} L(y ; \theta)=\frac{d}{d \theta} \log P(I(y) ; \theta)=\int_{I(y)} \frac{(x-\theta)}{\sigma^{2}} \phi\left(x \mid y ; \bar{\theta}, \sigma^{2}\right) d x
$$

as

$$
r^{*}=\left.\mathrm{E}\left(\frac{d}{d \theta} L(Y ; \theta)\right)\left(\frac{d}{d \theta} L(Y ; \theta)\right)\right|_{\theta=\theta^{*}}
$$

Equivalently, we can write, taking into account (16) with $N=1$,

$$
r^{*}=\mathrm{E}\left[Q^{\prime}\left(Y ; \theta^{*}, \theta^{*}\right) Q^{\prime}\left(Y ; \theta^{*}, \theta^{*}\right)\right],
$$


or as

$$
r^{*}=\sum_{k \in K} P\left(I_{k} ; \theta^{*}\right)\left(\int_{I_{k}} \frac{\left(x-\theta^{*}\right)}{\sigma^{2}} \phi\left(x \mid k h ; \theta^{*}, \sigma^{2}\right) d x\right)^{2} .
$$

Note that in the limiting case, when $h$ tends to 0 , we get $r^{*}=\sigma^{-2}=\left(\sigma^{*}\right)^{-2}$, as expected. It is easy to see that in any case the loss in information due to quantization decreases the Fisher information, i.e

$$
r^{*} \leq \sigma^{-2}
$$

3. A randomized $E M$-method. The integrals on the right hand side of (14) are expectations with respect to a conditional Gaussian density, and it is therefore natural to approximate them using a Markov Chain Monte Carlo (MCMC) algorithm, see $[9,12]$. A combination of the latter with the $E M$-algorithm leads to a stochastic approximation scheme called a randomized $E M$-method, first presented in $[4,5])$. A similar method has been developed independently for the problem of log-linear regression in [13].

The MCMC method. Thus to compute $\int_{I_{k}} x \phi\left(x \mid k h ; \bar{\theta}, \sigma^{2}\right) d x$ we generate an ergodic Markov chain $\bar{\xi}_{t}^{k}(\bar{\theta})$ on the state-space $I_{k}$, which is an interval of length $h$ (in case of no saturation), such that its invariant measure is $\phi\left(x \mid k h ; \bar{\theta}, \sigma^{2}\right)$ or $\phi\left(x \mid I_{k} ; \bar{\theta}, \sigma^{2}\right)$. For this purpose we use the Metropolis-Hastings method, with unnormalized target density

$$
\tau(x)=\tau(x, \bar{\theta})=e^{-(x-\bar{\theta})^{2} /\left(2 \sigma^{2}\right)} \chi_{I\left(y_{k}\right)}(x) .
$$

Let the initial transition kernel for the Metropolis-Hastings method be $q(x, y)=1 / h$ for all $x, y \in I\left(y_{k}\right)$, i.e. let the initial chain be simply an i.i.d. sequence with uniform distribution. Then we have a classic Metropolis algorithm defined by the acceptance probabilities

$$
\alpha(x, y ; \bar{\theta})=\min \left\{\frac{\tau(y, \bar{\theta})}{\tau(x, \bar{\theta})}, 1\right\}=\min \left\{e^{\frac{-(y-x)(y+x-2 \bar{\theta})}{2 \sigma^{2}}}, 1\right\} .
$$

For the generation of $\bar{\xi}_{\ell}^{k}(\bar{\theta})$ we will need an i.i.d sequence of random vectors $\left(U_{l}, V_{l}\right), l \geq 1$ with uniform distribution on $[0,1] \times[0,1]$, independent also of the initial state $\bar{\xi}_{0}^{k}(\bar{\theta})=\bar{\xi}_{0}^{k}$. The first component, $U_{l}$, is used to generate the next sate of the initial chain, while the second component, $V_{l}$, is used to realize the acceptance or rejection. We will thus use the following shorthand notation for the generation of $\bar{\xi}_{\ell}^{k}(\bar{\theta})$ :

The frozen parameter Markov chain on $I_{k}$ :

$$
\bar{\xi}_{\ell+1}^{k}(\bar{\theta})=F\left(\bar{\xi}_{\ell}^{k}(\bar{\theta}), U_{l+1}, V_{l+1} ; \bar{\theta}\right)
$$


Here $F$ depends on $\bar{\theta}$ via the acceptance probability $\alpha(x, y ; \bar{\theta})$.

Let $K_{N}$ be the set indices of quantization domains that show up in the observation sequence of length $N$. Let $k \in K_{N}$ and let the current state of the corresponding Markov chain be $\bar{\xi}_{\ell}^{k}(\bar{\theta})$. Then for large $L$ a good approximation of (14) is given by

$$
\theta=\sum_{k \in K_{N}} \frac{N_{k}}{N} \frac{1}{L} \sum_{\ell=1}^{L} \bar{\xi}_{\ell}^{k}(\bar{\theta}) .
$$

Allowing time-variation. When the above approximation is applied in an EMiteration it is reasonable to run the $E M$-algorithm and the $M C M C$ method in parallel. Let us now write $\bar{\theta}=\theta_{t}$, with $\theta_{t}$ still to be specified, and consider the time-varying Markovian dynamics

$$
\xi_{t+1}^{k}=F\left(\xi_{t}^{k}, U_{t+1}, V_{t+1} ; \theta_{t}\right)
$$

Here $\theta_{t}$ is the current approximation of the the maximum-likelihood estimator $\hat{\theta}_{N}$, which is in turn updated by an approximation of (30) as follows:

$$
\theta_{t+1}=\sum_{k \in K_{N}} \frac{N_{k}}{N} \frac{1}{t+1} \sum_{m=1}^{t+1} \xi_{m}^{k} .
$$

The above algorithm, defined by (31) and (32) is called a randomized EM-method. A simple calculation shows that (32) can be written in the following recursive form:

A randomized EM-method:

$$
\theta_{t+1}=\theta_{t}+\frac{1}{t+1} \sum_{k \in K_{N}} \frac{N_{k}}{N}\left(\xi_{t+1}^{k}-\theta_{t}\right)
$$

Let us stress again that the number of observations is fixed, and $\theta_{t}$ is expected to converge to $\hat{\theta}_{N}$, rather than to $\theta^{*}$.

4. A real-time recursive randomized $E M$-method. Consider now the situation when $N$ is not fixed, instead we have a flow of data, and for each new measurement the estimator of $\theta^{*}$ will be updated. Since, for increasing $N$, every integer $k$ will eventually occur in the observation sequence, we would need to generate an infinite number of Markov-chains. This is not practical, and also inconvenient for theoretical analysis. In particular the verification of the geometric ergodicity of the resulting Markov chain, as required in Part II, [1], would be difficult. Hence we confine ourselves to the case of quantization with saturation. The price of this is that the state space is non-compact, and the generation of the $M C M C$ method below and above the saturation level requires extra care. We shall not discuss this issue in this paper, since the verification of the conditions required for the underlying Markov chain, as given in Part II, [1], is not the subject of this paper. The quantization intervals will 
be denoted by $I_{k}$ as before with $k \in K$. If $|K|$ is large then it is unreasonable to update all the Markovian states at all time. Instead, at any time $T$, we update a single Markov chain, say $\bar{\xi}^{k}(\bar{\theta})$, where $k=k_{T}$ is the index of the current observation.

The first step is to modify the approximation to the $M$-step (30) so as to take into account the real time $T$. Let $N_{k, T}$ denote the number of visits to the domain $I_{k}$ up to time $T=N$, i.e. set

$$
N_{k, T}=\#\left\{n: x_{n} \in I_{k}, n \leq T\right\} .
$$

A convenient and reasonable approximation of (30) is obtained if we set $L=N_{k, T}$ for the quantization domain $I_{k}$, namely then (30) reduces to:

The M-step for increasing sample size:

$$
\theta=\frac{1}{T} \sum_{k \in K} \sum_{t=1}^{N_{k, T}} \bar{\xi}_{t}^{k}(\bar{\theta}) .
$$

Synchronization. To synchronize the internal times of the individual Markov chains $\bar{\xi}_{t}^{k}(\bar{\theta})$ let us define, for each $k$, a new, piecewise constant extension of $\bar{\xi}_{t}^{k}(\bar{\theta})$ as follows: first let $Z_{t}^{k}$ be the indicator of the event $x_{t} \in I_{k}$, i.e.

$$
Z_{t}^{k}=\chi_{I_{k}}\left(x_{t}\right)
$$

Define the new Markov chain $\bar{\xi}_{t}^{\circ, k}=\bar{\xi}_{t}^{\circ, k}(\bar{\theta})$ so that it stays constant at any time $t$, unless $x_{t} \in I_{k}$, and then follows the dynamics of $\bar{\xi}_{t}^{k}(\bar{\theta})$. Thus we get:

$$
\bar{\xi}_{t+1}^{\circ, k}=Z_{t}^{k} F\left(\bar{\xi}_{t}^{\circ, k}, U_{t+1}, V_{t+1} ; \bar{\theta}\right)+\left(1-Z_{t}^{k}\right) \bar{\xi}_{t}^{\circ, k} .
$$

Let the initial condition be $\bar{\xi}_{0}^{\circ, k}=\bar{\xi}_{0}^{k}$. Then $\left(\bar{\xi}_{t}^{\circ, k}, Z_{t}^{k}\right)$ is a Markov-process for each $k$, and so is

$$
\left(\bar{\xi}_{t}^{\circ}, Z_{t}\right)=\left(\bar{\xi}_{t}^{\circ, k}, Z_{t}^{k}\right), \quad k \in K .
$$

Also, the processes $\left(\bar{\xi}_{t}^{\circ, k}, Z_{t}^{k}\right)$ are independent as $k$ varies. Thus we can write the $M$-step (35) as

The M-step for the synchronized Markov-chain:

$$
\theta=\sum_{k \in K} \frac{1}{T} \sum_{t=1}^{T} Z_{t}^{k} \bar{\xi}_{t}^{\circ k}(\bar{\theta}),
$$

or, in an equivalent form, as

$$
0=\sum_{k \in K} \frac{1}{T} \sum_{t=1}^{T} Z_{t}^{k}\left(\bar{\xi}_{t}^{\circ k}(\bar{\theta})-\theta\right) .
$$


The context of the BMP-scheme. To put the above formula into the context of the BMP-scheme, see Chapter 1.1, Part II of [1], let us consider a function $H(Z, \xi ; \theta)$ defined over the state-space of the Markov-chain $\left(\bar{\xi}_{t}^{\circ}, Z_{t}\right)$ defined by

$$
H\left(Z, \xi^{\circ} ; \theta\right)=\sum_{k \in K} Z^{k}\left(\xi^{\circ, k}-\theta\right) / \sigma^{2} .
$$

Setting $Z=Z_{t}$ and $\xi^{\circ}=\bar{\xi}_{t}^{\circ}(\bar{\theta})$ we get a random field depending both on $(\theta, \bar{\theta})$ and $t$ :

$$
G_{t}(\theta, \bar{\theta})=\sum_{k \in K} Z_{t}^{k}\left(\bar{\xi}_{t}^{\circ, k}(\bar{\theta})-\theta\right) / \sigma^{2} .
$$

It is easy to see that, assuming stationary initialization for $\bar{\xi}_{t}^{\circ}(\bar{\theta})$, we have

$$
Q^{\prime}(\theta, \bar{\theta})=\frac{\partial}{\partial \theta} Q(\theta, \bar{\theta})=\mathrm{E}_{\xi} G_{t}(\theta, \bar{\theta}) .
$$

and thus for the asymptotic $Q$-function we have $\bar{Q}^{\prime}(\theta, \bar{\theta})=\mathrm{E} G_{t}(\theta, \bar{\theta})$. Noting that $\bar{Q}^{\prime}(\theta, \theta)=\frac{d}{d \theta} \bar{L}(\theta)$, see (20), it follows that the asymptotic problem of determining $\theta^{*}$ can be formulated as solving the equation

$$
\mathrm{E} G_{t}(\theta, \theta)=0,
$$

and thus the context needed for the BMP theory has been established.

To get a real-time randomized $E M$-method we proceed in the usual manner: let $\hat{\theta}_{t}$ be the estimate of $\theta^{*}$ at time $t$. Then generate the next state of a non-homogeneous Markov chain $\left(\xi_{t+1}^{\circ, k}\right)$ by

$$
\xi_{t+1}^{\circ, k}=Z_{t}^{k} F\left(\xi_{t}^{k}, U_{t+1}, V_{t+1} ; \hat{\theta}_{t}\right)+\left(1-Z_{t}^{k}\right) \xi_{t}^{\circ, k} .
$$

To update $\hat{\theta}_{t}$ we use a stochastic gradient method to maximize $\bar{L}(\theta)$. First we estimate the gradient $\frac{d}{d \theta} \bar{L}\left(\hat{\theta}_{t}\right)=\bar{Q}^{\prime}\left(\hat{\theta}_{t}, \hat{\theta}_{t}\right)$ by $G_{t+1}\left(\hat{\theta}_{t}, \hat{\theta}_{t}\right)$, which in turn is estimated on-line, see (40), by

$$
H\left(Z_{t+1}, \xi_{t+1}^{\circ}, \hat{\theta}_{t}\right)=\sum_{k \in K} Z_{t+1}^{k}\left(\xi_{t+1}^{\circ, k}-\hat{\theta}_{t}\right) / \sigma^{2}=\left(\xi_{N_{k^{\prime}, t+1}^{\prime}}^{k^{\prime}}-\hat{\theta}_{t}\right) / \sigma^{2},
$$

where $k^{\prime}=k_{t+1}^{\prime}$ is the index observed at time $t+1$. The definition of the nonhomogenous Markov chain $\xi_{t}^{k}$ is self-explanatory. With this notation a real-time randomized $E M$-method can be written as follows:

$A$ real-time randomized $E M$-method:

$$
\hat{\theta}_{t+1}=\hat{\theta}_{t}+\frac{\alpha}{t+1}\left(\xi_{N_{k^{\prime}, t+1}^{\prime}}^{k^{\prime}}-\hat{\theta}_{t}\right) / \sigma^{2},
$$

where $k^{\prime}=k_{t+1}^{\prime}$, and $\alpha>0$ is a step-size. In case of $\alpha=\sigma^{2}$, this is nothing else but the recursion for the arithmetic mean, and thus the following result is obtained: a real-time recursive randomized $E M$-procedure can be defined by

$$
\hat{\theta}_{T}=\frac{1}{T} \sum_{k \in K} \sum_{t=1}^{N_{k, T}} \xi_{t}^{k} .
$$


This method has been first presented in [6], but without justification for its convergence. The above derivation lends to a direct application of the BMP theory.

The associated ODE. The so-called associated ODE, see Chapter 1.5, Part II of [1], is obtained by fixing $\theta$ and taking expected value of the frozen parameter correction term in (44), assuming stationary initialization. Thus we get

$$
h(\theta)=\mathrm{E} \sum_{k \in K} Z_{t+1}^{k}\left(\bar{\xi}_{t+1}^{o, k}(\theta)-\theta\right) / \sigma^{2}=\mathrm{E} G_{t+1}(\theta, \theta)=\frac{d}{d \theta} \bar{L}(\theta),
$$

and the associated ODE is

$$
\dot{\theta}_{t}=\alpha h\left(\theta_{t}\right)=\alpha \frac{d}{d \theta} \bar{L}\left(\theta_{t}\right) .
$$

This is obviously asymptotically stable at $\theta=\theta^{*}$, thus a key technical condition of BMP theory, see Condition A7 of Chapter 1.6, Part II of [1], is, at least partially, satisfied.

The asymptotic variance. The asymptotic variance of the estimator $\hat{\theta}_{t}$ can be obtained using the algebra of Theorem 13, Chapter 4.5.3, Part II of [1] summarized subsequently under (62)-(65). This general result takes a particularly simple form if we choose

$$
\alpha=\left(r^{*}\right)^{-1}=-\left(\left.\frac{d^{2}}{d \theta^{2}} \bar{L}(\theta)\right|_{\theta=\theta^{*}}\right)^{-1}
$$

to be the inverse of the Fisher information for the problem of estimating $\theta^{*}$. Thus we get a stochastic Newton-method: the associated ODE is

$$
\dot{\theta}_{t}=\left(r^{*}\right)^{-1} h\left(\theta_{t}\right)=\left(r^{*}\right)^{-1} \frac{d}{d \theta} \bar{L}\left(\theta_{t}\right),
$$

with the Jacobian of the right hand side at $\theta=\theta^{*}$ being -1 , see also the paragraph following (63) below.

For a standard recursive maximum-likelihood method in stochastic Newton form we would get that the asymptotic covariance of the estimator is equal to the inverse of the Fisher information. In our case an additional source of randomness is that the score functions $\frac{d}{d \theta} L\left(y_{t} ; \hat{\theta}_{t}\right)$ are evaluated by an $M C M C$ method. Thus the asymptotic covariance of $\hat{\theta}_{t}$ is expected to be larger than the inverse of the Fisher information, and, assuming the validity of Theorem 13, Chapter 4.5.3, Part II of [1], is given as follows:

The asymptotic variance of $\hat{\theta}_{t}$ :

$$
\Sigma\left(\theta^{*}\right)=S\left(\theta^{*}\right)=\sum_{\tau}\left(r^{*}\right)^{-1} \mathrm{E}\left[H\left(Z_{t+\tau}, \bar{\xi}_{t+\tau}^{\circ}\left(\theta^{*}\right), \theta^{*}\right) \cdot H\left(Z_{t}, \bar{\xi}_{t}^{\circ}\left(\theta^{*}\right), \theta^{*}\right)\right]\left(r^{*}\right)^{-1} .
$$

The estimation of $r^{*}$. To estimate $r^{*}$ note that, by (25),

$$
r^{*}=\mathrm{E}\left[Q^{\prime}\left(Y ; \theta^{*}, \theta^{*}\right) Q^{\prime}\left(Y ; \theta^{*}, \theta^{*}\right)\right],
$$


and here, using (41),

$$
\bar{Q}^{\prime}\left(\theta^{*}, \theta^{*}\right)=\mathrm{E}_{\xi} G_{t}\left(\theta^{*}, \theta^{*}\right) .
$$

Taking into account the definition of $G$, see (40), we get

$$
r^{*}=r\left(\theta^{*}\right)=\mathrm{E}\left[\sum_{k \in K} Z_{t}^{k}\left(\bar{\xi}_{t}^{\circ, k}\left(\theta^{*}\right)-\theta^{*}\right) / \sigma^{2} \cdot \sum_{l \in K} Z_{t}^{l}\left(\bar{\eta}_{t}^{\circ, l}\left(\theta^{*}\right)-\theta^{*}\right) / \sigma^{2}\right],
$$

where $\bar{\eta}^{\circ, l}\left(\theta^{*}\right)$ is an independent copy of $\bar{\xi}^{\circ, k}\left(\theta^{*}\right)$. Letting $k^{\prime}$ be the observed index at time $t$, i.e. assuming $y \in I_{k^{\prime}}$. Then all terms, except those with indices $k=l=k^{\prime}$ are 0 , while $Z_{t}^{k^{\prime}}=1$, thus we get

$$
r^{*}=\mathrm{E}\left[\left(\bar{\xi}_{t}^{\circ, k^{\prime}}\left(\theta^{*}\right)-\theta^{*}\right) \cdot\left(\bar{\eta}_{t}^{\circ, k^{\prime}}\left(\theta^{*}\right)-\theta^{*}\right)\right] / \sigma^{4},
$$

A stochastic Newton method. Thus $r^{*}$ can be estimated recursively, and thus we get a fully adaptive stochastic Newton method:

$$
\begin{aligned}
& \hat{\theta}_{t+1}=\hat{\theta}_{t}+\frac{\hat{r}_{t}^{-1}}{t+1}\left(\xi_{N_{k^{\prime}, t+1}}^{k^{\prime}}-\hat{\theta}_{t}\right) / \sigma^{2}, \\
& \hat{r}_{t+1}=\hat{r}_{t}+\frac{1}{t+1}\left(\xi_{N_{k^{\prime}, t+1}}^{k^{\prime}}-\hat{\theta}_{t}\right)\left(\eta_{N_{k^{\prime}, t+1}}^{k^{\prime}}-\hat{\theta}_{t}\right) / \sigma^{4},
\end{aligned}
$$

with self-explanatory notations. Its associated ODE is

$$
\begin{aligned}
& \dot{\theta}_{t}=r_{t}^{-1} h\left(\theta_{t}\right), \\
& \dot{r}_{t}=r\left(\theta_{t}\right)-r_{t},
\end{aligned}
$$

with $r(\theta)$ defined implicitly in (50). The Jacobian matrix of the right hand side at the equilibrium point $\theta=\theta^{*}, r=r^{*}$ is of the form

$$
A=\left(\begin{array}{cc}
-1 & 0 \\
s & -1
\end{array}\right)
$$

which is obviously stable. Moreover it is partially stochastic Newton with respect to $\theta: A$ is block-triangular in a trivial manner, and its $(1,1)$-element is -1 .

The asymptotic variance of $\hat{\theta}_{t}$. The question arises whether the replacement of $r^{*}$ by $\hat{r}_{t}$ effects the asymptotic variance of $\hat{\theta}_{t}$. To answer this we compute the asymptotic covariance matrix of the combined estimator process $\left(\hat{\theta}_{t}, \hat{r}_{t}\right)$ denoted by, say $\Sigma=\Sigma\left(\theta^{*}, r^{*}\right)$. Taking into account the algebra of Theorem 13, Chapter 4.5.3, Part II of [1], summarized below under (62)-(65), we get that the $2 \times 2$ asymptotic covariance matrix $\Sigma=\Sigma\left(\theta^{*}, r^{*}\right)$ would satisfy the Lyapunov-equation

$$
\bar{A} \Sigma+\Sigma \bar{A}^{T}+S\left(\theta^{*}, r^{*}\right)=0
$$

with $\bar{A}=A+I / 2$ and $S\left(\theta^{*}, r^{*}\right)$ being defined in analogy with $S\left(\theta^{*}\right)$, see (48). Thus, in particular, $S_{11}\left(\theta^{*}, r^{*}\right)=S\left(\theta^{*}\right)$. The solution $\Sigma$ can be obtained via the integral 
representation, see $(64)$, as

$$
\Sigma=\int_{0}^{\infty} e^{\bar{A} t} S\left(\theta^{*}, r^{*}\right) e^{\bar{A}^{T} t} d t
$$

Taking into the block lower triangular structure of $A$ we get that $e^{A t}$ itself is block lower triangular, and its first block row is of the form $\left(e^{-t I / 2}, 0\right)$. Thus we get:

The asymptotic variance of $\hat{\theta}_{t}$ :

$$
\Sigma_{11}=\Sigma_{11}\left(\theta^{*}, r^{*}\right)=S_{11}\left(\theta^{*}, r^{*}\right)=S\left(\theta^{*}\right)=\Sigma\left(\theta^{*}\right) .
$$

We conclude that due to the fact that the combined estimation of $\theta^{*}$ and $r^{*}$ is partially stochastic Newton with respect to $\theta$, the asymptotic variance of $\hat{\theta}_{n}$ is not effected by the inclusion of $\hat{r}_{n}$.

Finally, a reduction of the effect of the additional randomness introduced by the $M C M C$ on the asymptotic variance of $\hat{\theta}$, see (48), can be decreased by taking say $L>>1$ samples of $\xi^{o, k}$ at each new observation. This amounts to redefining the $H$ function, defined under $(39)$, by setting $\tilde{\xi}^{\circ}=\left(\xi^{\circ, k, 1}, \ldots \xi^{\circ, k, L}\right)$, and

$$
H\left(Z, \tilde{\xi}^{\circ} ; \theta\right)=\sum_{k \in K} Z^{k} \frac{1}{L} \sum_{i=1}^{L}\left(\xi^{\circ, k, i}-\theta\right) / \sigma^{2}
$$

5. Estimating the variance. Let us now return to our original model $y=$ $q\left(\theta^{*}+e\right)$ and consider now the case when $\sigma^{*}$, the variance of the additive noise is unknown. It is easy to see that the $M$-step of the $E M$-method leads to the following updating formulas

$$
\begin{aligned}
\theta & =\sum_{k \in K} \frac{N_{k}}{N} \int_{I_{k}} x \phi\left(x \mid k h ; \bar{\theta}, \bar{\sigma}^{2}\right) d x, \\
\sigma^{2} & =\sum_{k \in K} \frac{N_{k}}{N} \int_{I_{k}}(x-\theta)^{2} \phi\left(x \mid k h ; \bar{\theta}, \bar{\sigma}^{2}\right) d x .
\end{aligned}
$$

Notice in (54) that $\theta$ does not depend on $\sigma$, thus we can solve the above equations successively. Then in analogy with the estimation of the location parameter, we arrive at the following real-time, partially stochastic Newton, randomized $E M$-method (see $(51),(52))$ :

$$
\begin{aligned}
& \hat{\theta}_{t+1}=\hat{\theta}_{t}+\frac{\hat{r}_{t}^{-1}}{t+1}\left(\xi_{N_{k^{\prime}, t+1}}^{k^{\prime}}-\hat{\theta}_{t}\right) / \hat{\sigma}_{t}^{2}, \\
& \hat{r}_{t+1}=\hat{r}_{t}+\frac{1}{t+1}\left(\xi_{N_{k^{\prime}, t+1}^{k^{\prime}}}^{k^{\prime}}-\hat{\theta}_{t}\right)\left(\eta_{N_{k^{\prime}, t+1}}^{k^{\prime}}-\hat{\theta}_{t}\right) / \hat{\sigma}_{t}^{4}, \\
& \hat{\sigma}_{t+1}^{2}=\hat{\sigma}_{t}^{2}+\frac{1}{t+1}\left(\left(\xi_{N_{k^{\prime}, t+1}}^{k^{\prime}}-\hat{\theta}_{t+1}\right)^{2}-\hat{\sigma}_{t}^{2}\right),
\end{aligned}
$$


where the dynamics of the time-varying Markov-chain now depends both on $\hat{\theta}_{t}$ and $\hat{\sigma}_{t}^{2}$.

To convert the above procedure into a fully stochastic Newton-method we need to estimate the $2 \times 2$ Fisher information matrix, say $R^{*}$. As have been said above, numerical experiments have shown, that the off-diagonal elements of the Fisher information matrix are zero: $r_{21}^{*}=r_{12}^{*}=0$. The estimation $r_{22}$ can be carried out along the lines described above for $r_{11}$.

6. Adaptive shifting. The BMP-scheme in general. To start this section we provide a brief summary to the basics of the BMP-method, without going into much technical details, see Chapter 1.1, Part II of [1]. Let us write the algorithm of the previous section using this general formalism, by setting $X=(\xi, Z)$, and $\theta$ for what was $(\theta, r)$, as follows:

$$
\hat{\theta}_{t+1}=\hat{\theta}_{t}+\frac{1}{t} H\left(X_{t+1}, \hat{\theta}_{t}\right),
$$

where $\theta \in D \subset \mathbb{R}^{p}$, with $D$ open, is a parameter, $X_{t}$ is a non-homogeneous Markov chain defined by a time-varying kernel of the form

$$
P\left(X_{t+1} \in A \mid \mathcal{F}_{t}\right)=\Pi_{\hat{\theta}_{t}, \theta^{*}}\left(X_{t}, A\right) .
$$

The dependence of the kernel on the true parameter $\theta^{*}$ has been explicitly indicated for reasons that will become clear soon. Here $\mathcal{F}_{t}$ is the $\sigma$-field generated by the past of the Markov process up to time $n$, and $A$ is any Borel subset of the state-space. In our application, when $X=(\xi, Z)$, this dependence shows up in the generation of the i.i.d. data $Z=\left(Z^{k}\right)$. The frozen parameter process, generated by

$$
P\left(X_{t+1} \in A \mid \mathcal{F}_{t}\right)=\Pi_{\theta, \theta^{*}}\left(X_{t}, A\right) .
$$

assuming a (unique) stationary initialization, will be denoted by $\bar{X}_{t}\left(\theta, \theta^{*}\right)$. Let $H(X, \theta)$ be a function with $\operatorname{dim} H=\operatorname{dim} \theta=p$, and let us define

$$
h\left(\theta, \theta^{*}\right)=\mathrm{E} H\left(\bar{X}_{t}\left(\theta, \theta^{*}\right), \theta\right) .
$$

Then the asymptotic estimation problem is to solve $h\left(\theta, \theta^{*}\right)=0$ for $\theta$. It is assumed that the solution is $\theta=\theta^{*}$. This is assumed for any choice of problem, i.e. for any $\eta=\theta^{*}$. Thus

$$
h(\eta, \eta)=0 .
$$

for all $\eta \in D$. It is assumed that the associated ODE

$$
\dot{\theta}_{t}=h\left(\theta_{t}, \eta\right)
$$

is asymptotically stable at $\eta$ for all $\eta \in D$. In particular, we assume that

$$
A(\eta)=\frac{\partial}{\partial \theta} h(\theta, \eta)_{\mid \theta=\eta}
$$


is a stable matrix for all $\eta$. Then, under a set of additional technical conditions, such as resetting, see $[1,8], \theta_{t}$, generated by $(59),(60)$, will converge to $\theta^{*}$ with probability 1.

The asymptotic covariance matrix of $\hat{\theta}_{t}$. The asymptotic covariance matrix of $\hat{\theta}_{t}$ is obtained, under restrictive conditions, in Theorem 13, Chapter 4.5.3, Part II of [1]. (Note that the conditions of this Theorem are not realistic. However it is likely that a rigorous derivation can be obtained under realistic assumptions by the methods of [7].) To summarize the algebra of this theorem let $A$ be the Jacobian matrix of the associated ODE at $\theta=\theta^{*}$, and let $\bar{A}=A+I / 2$ be stable. Define $S\left(\theta^{*}\right)$ as the asymptotic covariance of the empirical means of $H\left(\bar{X}_{t}\left(\theta^{*}\right), \theta^{*}\right)$ when the window size tends to infinity. This can be also expressed as

$$
S=S\left(\theta^{*}\right)=\sum_{\tau} \mathrm{E}\left[H\left(\bar{X}_{t+\tau}\left(\theta^{*}\right), \theta^{*}\right) \cdot H^{T}\left(\bar{X}_{t}\left(\theta^{*}\right), \theta^{*}\right)\right] .
$$

Then the asymptotic covariance matrix of $\hat{\theta}$, denoted by $\Sigma=\Sigma\left(\theta^{*}\right)$ would satisfy the Lyapunov-equation

$$
\bar{A} \Sigma+\Sigma \bar{A}^{T}+S=0
$$

The solution $\Sigma$ can be also obtained as

$$
\Sigma=\int_{0}^{\infty} e^{\bar{A} t} S e^{\bar{A}^{T} t} d t .
$$

This general result takes a particularly simple form if the recursive estimation method is stochastic Newton, i.e. if $A=-I$, in which case we get

$$
\Sigma=S
$$

Reconfiguration of measurements. Assume now that it is possible to reconfigure the measurement in such a way that, with a fixed and known $\delta>0$, we can estimate $\theta^{*}(\delta)=\theta^{*}+\delta$, just like $\theta^{*}$, using the real-time algorithm

$$
\bar{\theta}_{t+1}=\bar{\theta}_{t}+\frac{1}{t} H\left(X_{t+1}, \bar{\theta}_{t}\right)
$$

with

$$
P\left(X_{t+1} \in A \mid \mathcal{F}_{t}\right)=\Pi_{\bar{\theta}_{t}, \theta^{*}+\delta}\left(X_{t}, A\right) .
$$

Note that the dynamics of $X_{t}$ has changed by changing the true parameter from $\theta^{*}$ to $\theta^{*}+\delta$. Then, under appropriate conditions, $\bar{\theta}_{t}$ will converge to $\theta^{*}+\delta$, with probability 1. 
Let the a priori known optimal target location with maximal Fisher information matrix be $\eta^{*}$. This would be reached for the shift $\delta^{*}=\eta^{*}-\theta^{*}$. The question arises if we can estimate $\delta^{*}$ adaptively, so as to maximize the asymptotic efficiency of the procedure. In our application, the target value for the location parameter $\theta^{*}$ would be of the form $k h+h / 2$, with some integer $k$, assuming that $\sigma^{*}$ is known. Now, for any tentative $\delta$ the right correction term to $\delta$ would be

$$
\delta^{*}-\delta=\eta^{*}-\theta^{*}-\delta=\eta^{*}-\theta^{*}(\delta),
$$

which will be approximated by $\eta^{*}-\bar{\theta}_{t}$. Thus, we arrive at the following updating scheme:

The adaptive shifting algorithm:

$$
\delta_{t+1}=\delta_{t}+\frac{1}{t+1}\left(\eta^{*}-\bar{\theta}_{t}\right)
$$

We combine this with (66), where the Markovian dynamics of $X_{t}$, given by (67), is now modified so that the shift is time-varying, $\delta=\delta_{t}$ :

$$
P\left(X_{t+1} \in A \mid \mathcal{F}_{t}\right)=\Pi_{\bar{\theta}_{t}, \theta^{*}+\delta_{t}}\left(X_{t}, A\right) .
$$

It is then expected that $\left(\bar{\theta}_{t}, \delta_{t}\right)$ converges to $\left(\eta^{*}, \delta^{*}\right)$ with probability 1.

The associated $O D E$. To convince ourselves of this, we shall restrict ourself to the analysis of the associated ODE. Let us fix $\bar{\theta}$ and $\delta$. Then, taking the expectation of the correction terms in (66) and (68), we get

$$
\begin{aligned}
& \dot{\bar{\theta}}_{t}=h\left(\bar{\theta}_{t}, \theta^{*}+\delta_{t}\right), \\
& \dot{\delta}_{t}=\eta^{*}-\bar{\theta}_{t} .
\end{aligned}
$$

Now $h(\eta, \eta)=0$ for all $\eta$ implies that

$$
A(\eta)=\frac{\partial}{\partial \theta} h(\theta, \eta)_{\mid \theta=\eta}=-\frac{\partial}{\partial \eta} h(\theta, \eta)_{\mid \eta=\theta} .
$$

Thus, letting $A^{*}=A\left(\eta^{*}\right)$, the Jacobian-matrix of the associated ODE (70) at $\left(\eta^{*}, \delta^{*}\right)$ equals

$$
\tilde{A}=\left(\begin{array}{cc}
A^{*} & -A^{*} \\
-I & 0
\end{array}\right) .
$$

The stability of this matrix can be established in the case of quantized linear regression, when

$$
A^{*}=\left(\begin{array}{cc}
-1 & 0 \\
s & -1
\end{array}\right) .
$$

We shall not discuss the stability issue in the general case, since we proceed to improve the algorithm by using a Newtonian update. 
The stochastic Newton case. Consider now the case when the recursive estimation method (59), (60) is stochastic Newton, for any choice of $\theta^{*}=\eta$, in particular $A^{*}=$ $-I$. The resulting method, using adaptive shifting will not be stochastic Newton, since $\tilde{A} \neq-I$. In order to reduce the asymptotic covariance matrix of $(\bar{\theta}, \delta)$, we multiply the pair of equations $(66),(68)$, by $-\tilde{A}^{-1}$, given by

$$
-\tilde{A}^{-1}=\left(\begin{array}{cc}
0 & I \\
-I & I
\end{array}\right) .
$$

Thus we get a pair of equations

$$
\begin{gathered}
\bar{\theta}_{t+1}=\bar{\theta}_{t}+\frac{1}{t}\left(\eta^{*}-\bar{\theta}_{t}\right) \\
\delta_{t+1}=\delta_{t}+\frac{1}{t+1}\left(-H\left(X_{t+1}, \bar{\theta}_{t}\right)+\left(\eta^{*}-\bar{\theta}_{t}\right)\right) .
\end{gathered}
$$

with $X_{t}$ generated by (69). The first equation is free of noise and $\bar{\theta}_{t}$ converges to the known limit $\eta^{*}$. Thus it makes sense to fix $\bar{\theta}_{t}=\eta^{*}$, and thus we arrive to the final algorithm:

The final algorithm for adaptive shifting:

$$
\begin{gathered}
\delta_{t+1}=\delta_{t}-\frac{1}{t+1} H\left(X_{t+1}, \eta^{*}\right), \\
P\left(X_{t+1} \in A \mid \mathcal{F}_{t}\right)=\Pi_{\eta^{*}, \theta^{*}+\delta_{t}}\left(X_{t}, A\right) .
\end{gathered}
$$

The peculiarity of this procedure is that in the kernel $\Pi_{\eta^{*}, \theta^{*}+\delta_{t}}$ the first subindex is fixed permanently at the target value $\eta^{*}$, and the problem is adapted to this value by shifting $\theta^{*}$.

The associated ODE. To compute the associated ODE, let us fix $\delta$. Then the Markovian dynamics defines the frozen parameter process $\bar{X}_{t}\left(\eta^{*}, \theta^{*}+\delta\right)$, and thus the associated ODE is:

$$
\dot{\delta}_{t}=-h\left(\eta^{*}, \theta^{*}+\delta_{t}\right)
$$

Obviously, the Jacobian-matrix of the right hand side at the equilibrium point $\delta=$ $\delta^{*}=\eta^{*}-\theta^{*}$ is $-I$, thus the procedure is stochastic Newton. It follows that the asymptotic covariance matrix of $\delta_{t}$ is equal to

$$
S\left(\eta^{*}\right)=\sum_{\tau} \mathrm{E}\left[H\left(\bar{X}_{t+\tau}\left(\eta^{*}\right), \eta^{*}\right) \cdot H\left(\bar{X}_{t}\left(\eta^{*}\right), \eta^{*}\right)^{T}\right]
$$

It is easy to see that the above argument can be extended to partial adaptation, when $\theta^{*}=\left(\theta_{1}^{*}, \theta_{2}^{*}\right)$, and a target value, say $\eta_{1}^{*}$ is prescribed for $\theta_{1}^{*}$ only. In this case we assume that (59)-(60) is partially stochastic Newton with respect to $\theta_{1}^{*}$, i.e. the Jacobian $A^{*}$ has the form

$$
A^{*}=\left(\begin{array}{cc}
-I & 0 \\
U & V
\end{array}\right) \text {. }
$$


7. Application to the quantized linear regression problem. Let us redefine (36) so as to include the true values and the variance explicitly in the definition of $\bar{\xi}_{t}^{\circ, k}$. Note that the true parameters $\left(\theta^{*},\left(\sigma^{*}\right)^{2}\right)$ enter only via the data $Z_{t}^{k}$ :

$$
\bar{\xi}_{t+1}^{\circ, k}=Z_{t}^{k}\left(\theta^{*},\left(\sigma^{*}\right)^{2}\right) \cdot F\left(\bar{\xi}_{t}^{\circ, k}, U_{t+1}, V_{t+1} ; \bar{\theta}, \bar{\sigma}^{2}\right)+\left(1-Z_{t}^{k}\left(\theta^{*},\left(\sigma^{*}\right)^{2}\right)\right) \cdot \bar{\xi}_{t}^{\circ, k}
$$

Then for the algorithm with partially adaptive shifting we would generate a process $\xi_{t+1}^{\circ, k}$ with time-varying dynamics such that $\bar{\theta}$ is fixed at its target value $\eta^{*}$, while $\theta^{*}$ is shifted:

$$
\xi_{t+1}^{\circ, k}=Z_{t}^{k}\left(\theta^{*}+\delta_{t},\left(\sigma^{*}\right)^{2}\right) \cdot F\left(\xi_{t}^{k}, U_{t+1}, V_{t+1} ; \eta^{*}, \hat{\sigma}_{t}^{2}\right)+\left(1-Z_{t}^{k}\left(\theta^{*}+\delta_{t},\left(\sigma^{*}\right)^{2}\right)\right) \cdot \xi_{t}^{\circ, k}
$$

This process would then be used in the following updating formula for the shift $\delta$, see (75), and $r$ and $\sigma^{2}$ :

Estimation of $\theta^{*}$ and $\left(\sigma^{*}\right)^{2}$ using partial adaptive shifting:

$$
\begin{aligned}
& \delta_{t+1}=\delta_{t}-\frac{\hat{r}_{t}^{-1}}{t+1}\left(\xi_{N_{k^{\prime}, t+1}}^{k^{\prime}}-\eta^{*}\right) / \hat{\sigma}_{t}^{2}, \\
& \hat{r}_{t+1}=\hat{r}_{t}+\frac{1}{t+1}\left(\xi_{N_{k^{\prime}, t+1}}^{k^{\prime}}-\hat{\theta}_{t}\right)\left(\eta_{N_{k^{\prime}, t+1}}^{k^{\prime}}-\hat{\theta}_{t}\right) / \hat{\sigma}_{t}^{4}, \\
& \hat{\sigma}_{t+1}^{2}=\hat{\sigma}_{t}^{2}+\frac{1}{t+1}\left(\left(\xi_{N_{k^{\prime}, t+1}}^{k^{\prime}}-\hat{\theta}_{t+1}\right)^{2}-\hat{\sigma}_{t}^{2}\right) .
\end{aligned}
$$

The above dynamics simplifies significantly if $\sigma^{*}$ is known. In this case the Markov processes $\xi^{k}$ can be pre-computed, and in fact, they can be eliminated altogether. Indeed, taking into account (53), and letting $L$ tend to infinity, we can write the updating formula for $\delta$ as follows:

Estimation of $\theta^{*}$ for known $\left(\sigma^{*}\right)^{2}$ using adaptive shifting:

$$
\delta_{t+1}=\delta_{t}-\frac{\left(r^{*}\right)^{-1}}{t+1} \sum_{k \in K} Z_{t}^{k}\left(\theta^{*}+\delta_{t},\left(\sigma^{*}\right)^{2}\right) \cdot w_{k}
$$

where the weights

$$
w_{k}=\int_{I_{k}} \frac{\left(x-\eta^{*}\right)}{\left(\sigma^{*}\right)^{2}} \phi\left(x \mid k h ; \eta^{*},\left(\sigma^{*}\right)^{2}\right) d x,
$$

and $r^{*}$ can be pre-computed. Thus adaptive shifting does not only reduce the asymptotic variance, but can result in significant saving in computational time.

Figure 3 shows the convergence of the real-time randomized $E M$-method with adaptive shift. The true, ill-conditioned system is given by

$$
\theta^{*}=0, \quad\left(\sigma^{*}\right)^{2}=0.05,
$$


and the target value for $\theta^{*}$ is $\eta^{*}=0.5$. In order to make use of the advantageous effect of adaptive shifting, a relatively small variance has been chosen (see discussion about the Fisher information in the introduction). Both parameters are estimated using the proposed stochastic Newton algorithm. As seen from the figure, the algorithm converges to the optimal shift

$$
\delta^{*}=\eta^{*}-\theta^{*}=0.5
$$

The algorithm was initialized with the parameters

$$
\hat{\delta}_{0}=0, \quad \hat{\sigma}_{0}^{2}=0.08 .
$$
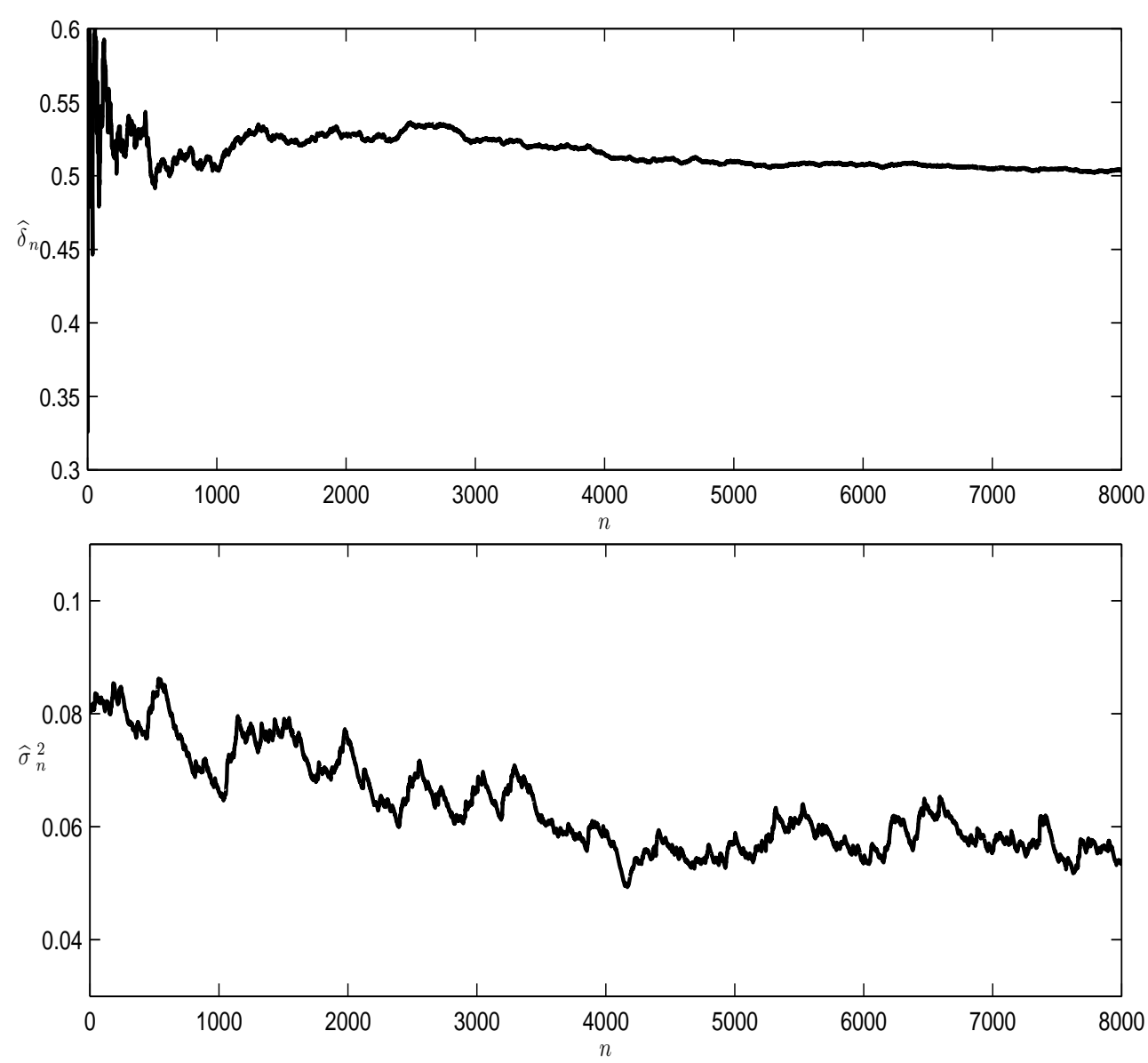

FIG. 3. The convergence of the estimated parameters.

To illustrate the superior performance of adaptive shifting, we compared the proposed method to the stochastic Newton-type real-time randomized $E M$-method without adaptive shift. As a figure of merit we used simple statistics about the estimator. We ran both algorithms 200 times for the same ill-conditioned system described above, 
TABLE 1

Performace comparision of the algorithms with and without adaptive shift.

\begin{tabular}{|l|l|l|}
\hline \multirow{2}{*}{} & \multicolumn{2}{|c|}{ Empirical mean } \\
\cline { 2 - 3 } & without shift & with shift \\
\hline$\hat{\theta}_{8000}$ & 0.001736 & -0.000136 \\
\hline$\hat{\sigma}_{8000}^{2}$ & 0.049004 & 0.055897 \\
\hline
\end{tabular}

\begin{tabular}{|l|l|l|}
\hline \multirow{2}{*}{} & \multicolumn{2}{|c|}{ Empirical variance } \\
\cline { 2 - 3 } & without shift & with shift \\
\hline$\hat{\theta}_{8000}$ & 0.000413 & 0.000051 \\
\hline$\hat{\sigma}_{8000}^{2}$ & 0.000048 & 0.001931 \\
\hline
\end{tabular}

each run had 8000 iterations, and calculated the empirical mean and variance of the estimator value after the last iteration. From Table 1 we can conclude that, with respect to the location parameter, the proposed adaptive shifting method is much more accurate than the algorithm without shift.

\section{REFERENCES}

[1] A. Benveniste, M. MÉtivier, And P. Priouret. Adaptive algorithms and stochastic approximations. Springer-Verlag, Berlin, 1990.

[2] R. Brockett and D. Liberzon. Quantized feedback stabilization of linear systems. IEEE Transactions on Automatic Control, 45(2000), pp. 1279-1289.

[3] A. P. Dempster, N. M. Laird, And D. B. Rubin. Maximum likelihood from incomplete data via the EM algorithm (with discussion). Journal of Royal Statistical Society, Series B., 39(1977), pp.1-38.

[4] L. Finesso, L. Gerencsér, And I. KMecs. Estimation of parameters from quantized noisy observations. In: Proc. of the European Control Conference, AM-3, F589, Karlsuhe, Germany, 1999.

[5] L. Finesso, L. Gerencsér, And I. Kmecs. A randomized EM-algorithm for estimating quantized linear gaussian regression. In: Proc. of the 38th IEEE Conference on Decision and Control. Phoenix, Arizona, USA, pages 5100-5101, 1999.

[6] L. Finesso, L. Gerencsér, And I. Kmecs. A recursive randomized EM-algorithm for estimation under quantization error. In: Proc. of the American Control Conference. Chicago, Illinois, USA, pages 790-791, 2000.

[7] L. Gerencsér. A representation theorem for the error of recursive estimators. Submitted to SIAM J. Control and Optimization, 44(2005), pp. 2123-2188.

[8] L. Gerencsér And Z. MÁtyás. Almost sure and $L_{q}$-convergence of the re-initialized BMP scheme. In: Proc. of the 46th IEEE Conference on Decision and Control. New Orleans, Louisiana, USA, 2007.

[9] J.M. Hammersley and D.C. Handscomb. Monte-Carlo methods. London: Methuen \& Co., 1967.

[10] E. Masry and S. CAmbanis. Signal identification after noisy nonlinear transformation. IEEE Transactions on Information Theory, IT-26(1980), pp.50-58.

[11] M. MÉtivier And P. Priouret. Application of a Kushner and Clark lemma to general classes of stochastic algorithms. IEEE Transactions on Information Theory, 30(1984), pp. 140-151.

[12] N. Metropolis, A. Rosenbluth, M. Rosenbluth, A. Teller, and E. Teller. Equations of state calculations by fast computing machines. J. Chem. Phys., 21(1953), pp. 1087-1091.

[13] V. Solo. Adaptive algorithms and Markov chain Monte Carlo methods. In: Proc. of the 38th IEEE Conference on Decision and Control. Phoenix, Arizona, USA, pages 1775-1778, 1999. 
[14] B. Widrow and I. Kollár. Quantization Noise in Digital Computation, Signal Processing, and Control. Cambridge University Press, 2008. 